\title{
Development of a rapid LATE-PCR-based dipstick for the detection of schistosomiasis haematobium in human urine samples
}

\begin{abstract}
Introduction: Schistosomiasis caused by Schistosoma haematobium has become a serious public health problem in tropical and subtropical countries. It is associated with considerable morbidity and mortality in developing and underdeveloped countries. Diagnosis of Schistosoma haematobium infection in both adult and children patients is a clinically important challenge. In the work presented here, we report on an alternative diagnostic method based on presence of the $S$. haematobium -specific Dra 1, 121 bp repeat fragment in human urine. This study aims to develop Linear-After-The-Exponential- Polymerase Chain Reaction (LATE-PCR) dipstick for the detection of Schistosoma haematobium in urine samples.
\end{abstract}

Methodology: In an effort to developed or improved the current PCR techniques LATE-PCR Lateral flow was developed, in order to allow specie-specific amplification of Schistosoma haematobium in urine samples.

Results: Evaluation study on 50 urine samples showed $100 \%$ sensitivity and specificity suggesting the primers and designed probe is specific. Sensitivity of LATE-PCR currently was said to be higher when compared to parasitological methods (Microscopy). LATE-PCR dipstick is highly sensitive since 30 positive urine samples with very low eggs burden were also seen to be positive by the test. The analytical sensitivity was performed and detection limit determine for Schistosoma haematobium. The detection limit of the PCR reaction on Schistosoma haematobium was found to be $1 \mathrm{fg} / \mu \mathrm{l}$ using 10 fold serial dilution.

Conclusion: LATE-PCR dipstick developed in this study provides a valuable alternative for the detection of Schistosoma haematobium infection in the study area to speed up diagnosis in addition to the conventional method currently used.

Keywords: Polymerase chain reaction; Lateral flow assay; Schistosoma haematobium; Urine samples
Volume 3 Issue I - 2017

\author{
Kabiru Mohammed,' Aziah Ismail, ${ }^{2}$ Julia \\ Omar, ${ }^{3}$ Ikeh E Ifeanyi, Joseph, Fabiyi P, ${ }^{5}$ Salisu \\ Ismail, ${ }^{6}$ Mohamad Rusli ${ }^{3}$ \\ 'Department of Medical Microbiology, Usmanu Danfodiyo \\ University, Nigeria \\ ${ }^{2}$ Department of Molecular Medicine, Universiti Sains Malaysia, \\ Malaysia \\ ${ }^{3}$ Department of Community Medicine, Universiti Sains Malaysia, \\ Malaysia \\ ${ }^{4}$ Department of Medical Microbiology, University of Jos, Nigeria \\ ${ }^{5}$ Department of Applied Parasitology, Usmanu Danfodiyo \\ University, Nigeria \\ ${ }^{6}$ Department of Surgery, Usmanu Danfodiyo University Teaching \\ Hospital, Nigeria
}

\section{Correspondence: Kabiru Mohammed, Department of Medical Microbiology, Faculty of Medical Laboratory Sciences, Usmanu Danfodiyo University, Sokoto State, Nigeria, Tel} +234(0)9053490544, Email kmohd1970@yahoo.co.uk

Received: June 28, 2017 | Published: September 06, 2017

\section{Introduction}

Schistosomiasis is a serious and significant public health problem affecting more than 200 million people in endemic regions worldwide. ${ }^{1}$ It was reported that there was increased in the spread of Schistosomiasis due to increase in off-track tourism which eventually result in increased number of people infected in non-endemic areas of the world. ${ }^{2,3}$ However, the majority of cases annually reported occur mainly in sub-Saharan Africa and out of this 150,000 deaths were attributed to Schistosoma haematobium infection. ${ }^{4}$ Haematuria is one of the commonest sign reported due to Schistosoma haematobium infection, while other important noticeable sign which may follow includes proteinuria and dysuria especially among school age children. Invention of modern drugs such as praziquantel has reduced the number of death due to kidney failure and other deformity reported as a result of Schistosoma haematobium infection. ${ }^{1,5,6}$ The infection occurs following contact with contaminated water containing cercariae, an infective stage of the parasite, during their daily activities such as irrigation farming, bathing, swimming, fishing and other recreational activities. The disease causes significant morbidity and mortality worldwide with highest peak prevalence and intensity of infection among 10-20 years age group Rambu et al. 2013. World Health Organization estimated that 235 million cases of schistosomiasis occur world -wide, with 732 million people at risk of infection and another 200,000 people died each year especially in subSaharan Africa where the disease is said to be endemic.?

Currently available methods for Schistosomiasis diagnosis are based on urine and stool examination for parasite eggs detection which often lack sensitivity and specificity while serological methods specificity is low and thus result in misdiagnosis of positive samples. Several journal articles reported the DNA based diagnostic tool by PCR which seems to have higher sensitivity and specificity Tandem repeat sequence of Schistosoma haematobium based on 121 base pairs was used in designing specific primers and amplification of corresponding sequence in human stool and serum samples. ${ }^{8-10}$

Several alternative efforts have been made in order to improve on microscopy by developing immunodiagnostic methods which make use of parasite-specific antibodies and circulating Antigen detection refer to as Cathodic Circulating Antigen (CCA). One advantages of the method is that it can detect current infection, however, it cannot be use in differentiating current and past infections. Antibody-based test have been shown to be more sensitive when compared with microscopy particularly in situations when egg counts are low but lack specificity. ${ }^{11,12}$ In order to control and evaluate Schistosomiasis infection pattern, it is thus necessary to establish or develop a new diagnostic approach that is simple, rapid (10-15 minutes). Microscopy is currently the gold standard for parasitological method of 
Schistosomiasis diagnosis as it is relatively simple and inexpensive. However, one short- coming with parasitological techniques is that of low sensitivity especially in endemic areas where prevalence and intensity of infection is very low, thus making the method less appropriate for post- treatment intervention as well as measurement of morbidity in low resource settings. ${ }^{13,14}$ Parasitological techniques cannot be used in diagnosis of infection at a prepatent period when the eggs are not yet being produced by the parasite in recent infection which is another set-back to this method of diagnosis (Figures $2 \&$ Figure 3).

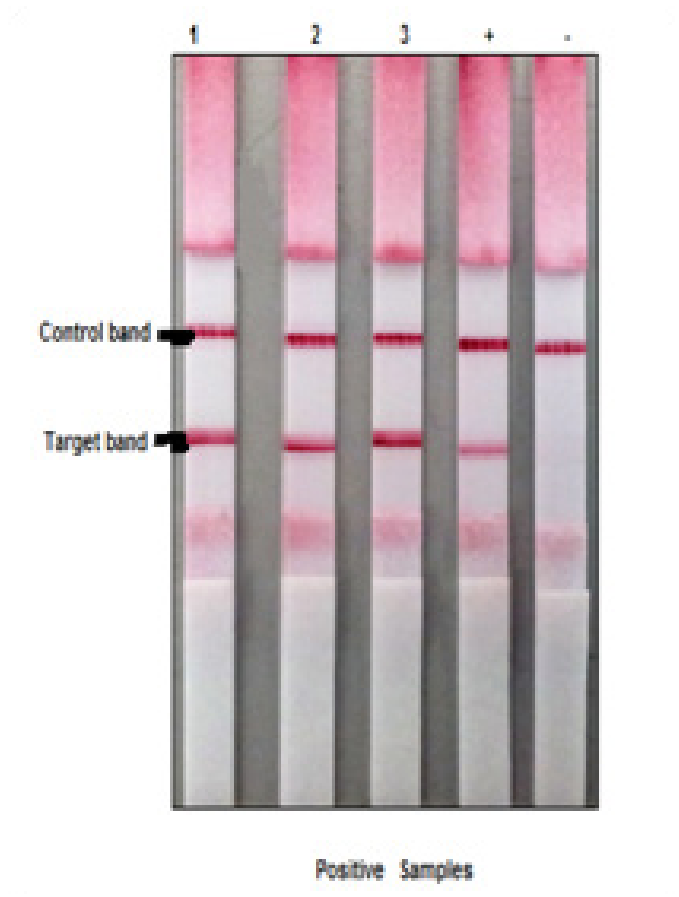

Figure I Positive urine samples.

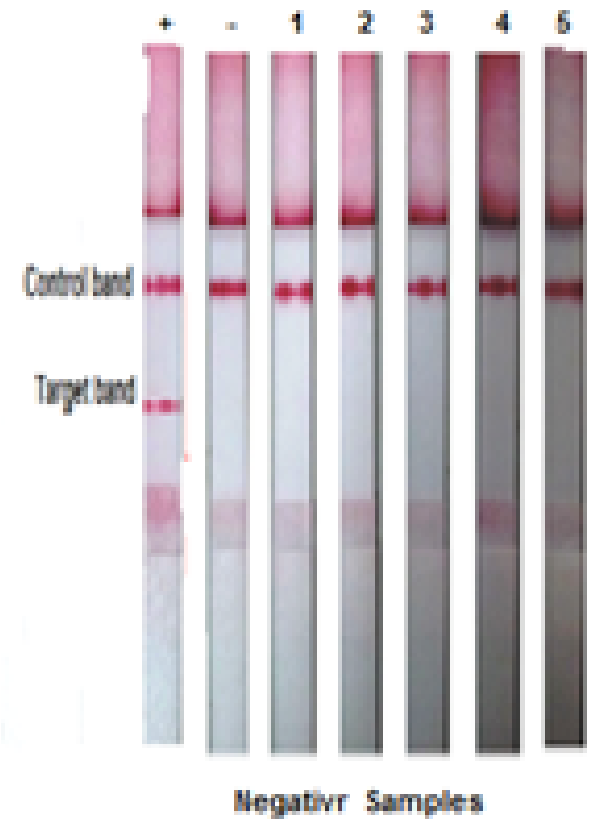

Figure 2 Negative urinal samples.

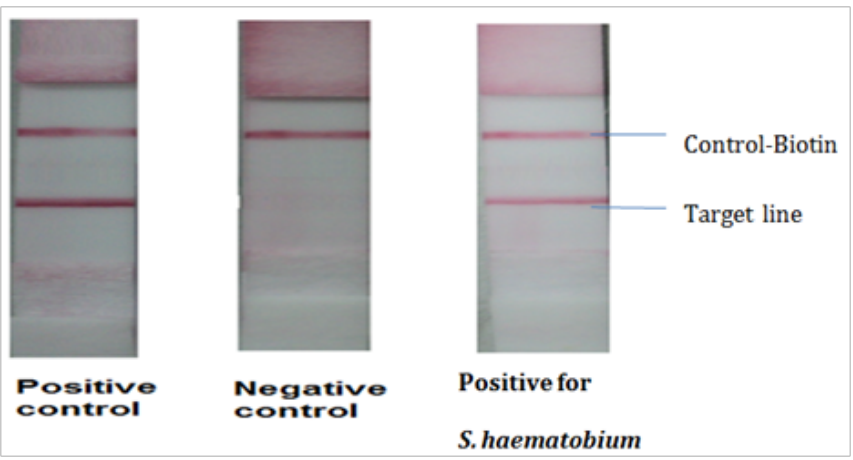

Figure 3 Showing positive control strip, negative control strip and positive for target S.haematobium.

\section{Materials and Methods}

\section{Materials}

Antibodies used: Control Line: anti-mouse IgG-biotin from Sigma Aldrich (St. Louis, USA).

Target line: Rabbit anti-digoxigenin IgG from Sigma Aldrich (St. Louis, USA).

Lateral flow buffer: 10mM Phosphate Buffered Saline ( $\mathrm{pH} 7.4$ ), $1 \%$ Bovine serum albumin, $0.5 \%$ Tween- 20 .

Gold-conjugate buffer: $2 \mathrm{mM}$ borate, $1 \%$ bovine serum albumin, $5 \%$ trehalose.

\section{Diagnostic test sample}

For evaluation of LATE-PCR dipstick sampling was done from 50 participants involved in the study. The positive urine samples were obtained from villages in north-western, Nigeria while the negative control samples (obtained from apparently healthy individuals) were obtained from Kubang Kerian Kelantan, Malaysia. The villages where the positive urine samples were obtained were endemic for Schistosoma haematobium and no mass treatment with Schistosomiasis had taken place in the study villages. The participants from the villages were given clean plastic bottle and were asked to produce urine samples and were subjected to microscopic analysis which reveals a large number of eggs from the samples. Within 24hours of samples collection they were preserved in $10 \%$ formalin and was refrigerated at $-20^{\circ} \mathrm{C}$ until further processing was performed at the institute of research in molecular medicine (INFORMM Malaysia).

\section{Methodology}

\section{Preparation of lateral flow strip}

The strip was constructed using sample pads, glass fibre conjugate pads, nitrocellulose membrane and cellulose absorbent pads and assembled onto a plastic adhesive backing card. Conjugate pad and absorbent pad were placed at opposite ends of the nitrocellulose membrane, overlapping the membrane by approximately $2 \mathrm{~mm}$ to allow the flow of liquid through the strip by capillary action. Biotin $(0.6 \mathrm{mg} / \mathrm{ml}$-neat $)$ was diluted at $1: 8$ and dispensed as a control line while anti-FITC $(6.7 \mathrm{mg} / \mathrm{ml}$-neat $)$ was diluted at $1: 16$ and dispensed as a target line onto the nitrocellulose membrane. The strip was then and dried at room temperature in drying cabinet with a relative humidity (RH) around $20-30 \%$ for 24 hours. 


\section{Preparation of dried streptavidin-gold conjugate}

The amount of $225 \mu 1$ of OD 20 streptavidin-gold conjugates were diluted into $675 \mu$ l of conjugate buffer to a final concentration of OD 5, dispensed onto a $15 \mathrm{~cm}$ of glass fibre conjugate pad and dried at room temperature in drying cabinet with a relative humidity $(\mathrm{RH})$ around $20-30 \%$ for 24 hours before strip assembly. The assembled card was cut into $5 \mathrm{~mm}$ test strips following the standard operating procedure of the strip cutter.

\section{Performing LATE-PCR LFA strip from amplified LATE-PCR product}

Onto the sample pad ten microliter of the LATE-PCR product was applied adjacent to the conjugate pad and 140ul of standard LFA running buffer (10mM PBS of $\mathrm{pH} 7.4,1 \% \mathrm{BSA}$ and $0.05 \%$ Tween-20 ] was placed onto the sample pad. The mixture migrated towards the absorbent pad by capillary attraction. The result can be visualized in the form of red lines by using naked eyes within fifteen minutes.

\section{Interpretation of LATE-PCR Dipstick LFA strip result}

Presence of all two lines (control, and target) along the strip indicates valid positive results. Presence of only one line at control (i.e control band only) indicates a valid negative result. Illustration of LATE-PCR LFA Strip results are shown in Figure 3 below.

\section{Primer design and PCR}

Sequences of primers used in this study were obtained from NCBI website and were blast (Basic local align tool for nucleotide search data base was used to check for specificity of the gene and also from Hamburger et al. ${ }^{9}$ Two sets of primers were designed to amplify 121 bp tandem repeat DNA sequences of and Schistosoma. haematobium. All the primers were synthesis by $1^{\text {st }}$ Base company.

\section{Extraction of parasite genomic DNA}

The extraction of genomic DNA was performed using a Nucleospin kit (Macherey-Nagel, Germany) at the Institute for Research in Molecular Medicine (INFORMM), Universiti Sains Malaysia, Health Campus Kelantan, Malaysia. The procedure for the extraction was done as provided by the manufacturer.

\section{PCR amplification}

PCR will be carried out using specific primers. The primers included both forward and reverse primers targeted for S. haematobium (DRA1 gene). A total volume of $25 \mu \mathrm{l}$ of PCR master mix will be prepared including 1X PCR buffer, $3.5 \mathrm{mM} \mathrm{MgCl}_{2}, 0.2 \mathrm{mM}$ dNTPs, $0.4 \mu \mathrm{M}$ forward and reverse primers, $2.5 \mathrm{U}$ Taq DNA polymerase and DNA template will be added into $200 \mu$ PCR tube. A negative control will be included in each run, which consisted of all the above mentioned mixture except the DNA template.

Amplification will be performed as follows: Initiation denaturation step at $95^{\circ} \mathrm{C}$ for 5 minutes and then followed by temperature cycling: denaturation at $95^{\circ} \mathrm{C}$ for 1 minute, annealing temperature at $55^{\circ} \mathrm{C}$ for 1 minute and extension at $72^{\circ} \mathrm{C}$ for 1 minute with total cycles of 35 , and final extension of $72^{\circ} \mathrm{C}$ for 10 minutes. Amplifications will be carried out in PTC-200 thermal cycler (MJ Research, USA) and 10 $\mu$ l of PCR products will be analyzed in $2 \%$ agarose gel stained with ethidium bromide $(10 \mathrm{mg} / \mathrm{ul})$ and visualized with UV light or via lateral flow assay.

\section{Results}

Evaluation study on 50 urine samples showed $100 \%$ sensitivity and specificity suggesting the primers and designed probe is specific without any cross- reaction with other microorganisms. The sensitivity of LATE-PCR was said to be higher when compared to Microscopy. LATE-PCR dipstick is highly sensitive since 30 positive urine samples with very low eggs burden were also seen to be positive by the test (Table 1). The analytical sensitivity was performed and detection limit determine for schistosoma haematobium. The detection limit of the PCR reaction on Schistosoma haematobium was found to be $1 \mathrm{fg} / \mu \mathrm{l}$ using 10 fold serial dilution.

Table I Evaluation study on 50 urine samples showed 100\% sensitivity and specificity suggesting the primers and designed probe is specific without any crossdetection with other microorganisms

\begin{tabular}{llll}
\hline \multirow{2}{*}{ Category } & \multicolumn{2}{l}{ Late-PCR dipstick for the detection of S. Haematobium } & Total \\
\cline { 2 - 4 } & Positive & Negative & \\
\hline Microscopy positive & 30 & 0 & $30\left({ }^{\mathrm{a}} 100 \%\right)$ \\
Microscopy negative & 0 & 20 & $20\left({ }^{\mathrm{b}} 100 \%\right)$ \\
& $30\left({ }^{\mathrm{a}} 100 \%\right)$ & $20\left({ }^{\mathrm{b}} 100 \%\right)$ & 50 \\
\hline
\end{tabular}

a: Sensitivity b: Specificity c: Positive predictive value (PPV) d: Negative predictive value (NPV)

The capture reagents used in the development of S.haematobium LATE-PCR dipstick, which were biotinylated anti-mouse IgG and monoclonal anti-FITC(anti-FITC). The concentration of each capture reagent was optimized in order to obtained the optimum intensity of the red line on the LFA strip. The optimization of the capture reagents was initiated using the control red line which is biotinylated antimouse IgG. The biotinylated anti-mouse IgG was diluted serially in $10 \mathrm{~m}$ M PBS ( $\mathrm{pH}$ 7.4). One microliter (ul) of each diluted biotinylated anti-mouse $\operatorname{IgG}$ was dispensed into the nitrocellulose membrane and dried in drying cabinet for 24 hours with RH $20 \%$, to 30\%. An amount of $140 \mathrm{ul}$ of a running buffer was dispensed onto the sample pad and then allowed to migrate towards the absorbent pad for 15 minutes. The intensity of red line was then visualized.

\section{Discussion}

Schistosomiasis is one of the parasitic diseases with highest morbidity and mortality in Sub-Saharan Africa and second to none in terms of prevalence of infection among water- borne diseases in developing countries in Africa, middle east, Caribbean and Asia. ${ }^{15,16}$ According to an estimate of World Health Organisation's 700 million people are at risk of acquiring the infection in 76 countries while more than 207 million people are ill with Schistosomiasis, $85 \%$ of whom lived in Sub-Saharan Africa. ${ }^{15,17}$ Microscopy which is currently the gold standard for parasitological method of schistosomiasis diagnosis as it is relatively simple and inexpensive. However, one short coming with parasitological techniques is that of insensitivity especially in 
endemic areas where prevalence and intensity of infection is very low, thus making the method less appropriate for post treatment intervention as well as measurement of morbidity in low resource settings. ${ }^{13,14}$ Parasitological techniques cannot be used in diagnosis of infection at a prepatent period when the eggs are not yet being produce by the parasite in recent infection which is another set-back to this method of diagnosis. Consequently there is need to development of an updated sensitive, rapid and specific diagnostic tools which has the potential to be used in the routine diagnosis of schistosomiasis due to schistosoma haematobium.

In an effort to develop an alternative diagnostic tool for Schistosoma haematobium diagnosis a primer pairs was designed specifically for the detection of schistosoma haematobium in human urine samples. Thus a single PCR amplicon of the parasite DNA can be detected with limits of detection ranging from ifg/ $\mu$ l. Similar results were found by Pontes et al. ${ }^{8}$ with highly repetitive sequence in the parasite genome. The result of our finding showed that Schistosoma haematobium was found to be endemic in the study area of North-western, Nigeria. In an attempt to overcome short comings of the current diagnostic methods for detection of schistosomiasis haematobium, we have developed a laboratory tested LATE-PCR dipstick that may be suitable for this purpose.

Since the LATE-PCR dipstick assay was based on detection and amplification of a highly repeated DNA sequence. LATE-PCR dipstick being highly sensitive than the conventional PCR and this is demonstrated by the detection limit of as low as $1 \mathrm{fg} / \mathrm{ul}$ of S.haematobium in urine sample. With regards to specificity of the present test The amplification reaction showed to be specific without any cross reaction with DNA from other UTI micro-organism The LATE-PCR dipstick assay enable identification of $S$. haematobium as long as the DNA of the organisms remain intact in the sample to be tested. The present Study showed that the developed LATEPCR dipstick is a promising tool for the specific, sensitive and rapid diagnosis of infection with schistosoma haematobium It is advice that future research studies on the application of this DNA based assay should be carried out with other schistosome specie and with the hope of evaluating its applicability in the field as well as in the follow up of chemotherapy.

\section{Conclusion}

The present study findings revealed that LATE-PCR dipstick presented a high sensitivity and specificity in low endemic areas and therefore could serve as a valuable alternative method for diagnosis of Schistosomiasis haematobium infection in underdeveloped as well as in developing countries. ${ }^{18,24}$ There is the needs to evaluate and applied this DNA based assay as a screening tool in areas of low endemicity because of the techniques being simple, rapid, sensitive and equipment free assay.

\section{Acknowledgements}

None.

\section{Conflict of interest}

The author declares no conflict of interest.

\section{References}

1. Gryseels B, Polman K, Clerinx J, et al. Human schistosomiasis. Lancet. 2006;368(9541):1106-1118.
2. Chitsulo L, Engels D, Montresor A, et al. The global status of schistosomiasis and its control. Acta Trop. 2000;77(1):41-51.

3. Sandoval N, Siles-Lucas M, Pérez-Arellano JL, et al. A new PCRBased approach for the specific amplification of DNA from different Schistosome specie applicable to human urine samples. Parasitology. 2006;133(Pt 5):581-587.

4. Van Der Werf MJ, De Vlas SJ, Brooker S, et al. Quantification of clinical morbidity asso-ciated with schistosome infection in sub-Saharan Africa. Acta Trop. 2003;86(2-3):125-139.

5. King $\mathrm{CH}$. Parasites and Poverty, the case of schistosomiasis. Acta Tropica. 2010;113(2):95-104.

6. Koukounari A, Webster JP, Donnelly CA, et al. Sensitivities and Specificities of Diagnostic tests and infectious prevalence of S.haematobium estimated from data on adults in villages in Northwestern Accra, Ghana. Am J Trop Med Hyg. 2011;80(3):435-441.

7. Alebie G, Erko B, Aemero M, et al. Epidemiological study on schistosoma mansoni infections in Sanja area, Amhara region,Ethiopia. Parasites and Vector. 2014;7:15.

8. Pontes LA, Oliveira MC, Katz N, et al. Comparison of a polymerase chain reaction and the Kato-Katz technique for diagnosing infection with Schistosoma mansoni. Am J Trop Med Hyg. 2003;68(6):652-656.

9. Hamburger J, Turetski T, Kapeller I, et al. Highly repeated short DNA sequences in the genome of Schistosoma mansoni recognized by a species-specific probe. Mol Biochem Parasitol.1991;44(1):73-80.

10. Hamburger J, Turetski T, Kapeller I, et al. Highly repeated short DNA sequences in the genome of Schistosoma mansoni recognized by a species-specific probe. Mol Biochem Parasitol. 1991;44(1):73-80.

11. Doenhoff MJ, Butterworth AE, Hayes RJ, et al. Epidemiology and serodiagnosis of Schistosomiasis in Kenya using crude and purified egg antigens of Schistosoma mansonii ELISA. Trans R Soc Trop Med Hyg. 1993;87(1):42-48.

12. Nausch N, Dawson EM, Midzi N, et al. Field evaluation of a new antibody-based diagnostic for Schistosoma haematobium and $\mathrm{S}$. mansoni at the point of care in North-east Zimbabwe. BMC Infect Dis. 2014;14:165

13. Hagag SH, Abdullah SM. Molecular diagnosis of schistosomiasis mansoni. Life Science Journal. 2011;8(2):395-404.

14. WHO. Control of Schistosomiasis: Report of a WHO Expert Committee. Switzerland: Technical Report Series; 1994. 830 p.

15. Dawet A, Longmut R, Benjamin CB, et al. Prevalence and intensity of Schistosomiasis haematobium among resident of Gwong and Kabong in Jos North Local Government Area, Plateau State, Nigeria. International Journal of Biol Chem Sci. 2012;6(4):1557-1563.

16. Wilson R, Rollinson D, Simpson AG. Cercariae to liver worms: development and migration in the mammalian host. In: The Biology of Schistosomes from Genes Ta Latrines, England: Acadamic Press; 1987. p. $115-146$.

17. First WHO report on neglected tropical diseases working to overcome the global impact of neglected tropical diseases. Switzerland: World Health Orga-nization; 2010.

18. Gryseels B, Stelma FF, Talla I, et al. Epidemiology, immunology and chemotherapy of Schistosoma mansoni infection in a recently exposed community in Senegal. Trop Geogr Med. 1994;46(4 Spec No):209-219.

19. Hamburger J, He-Na, Abbasi I, et al. Polymerase chain reaction assay based on a highly repeated sequence of Schistosoma haematobium: a potential tool for monitoringschistosome-infested water. Am J Trop Med Hyg. 2001;65(6):907-911. 
20. Kato-Hayashi N, Kirinoki M, Iwamura Y, et al. Identification and differentiation of human Schistosomes by PCR. Exp Parasitol. 2010;124(3):325-329.

21. Leder K, Weller P. Epidemiology, pathogenesis, and clinical features of schistosomiasis; 2009.

22. WHO. Prevention and control of schistosomiasis and Soil transmitted helminthiasis. Switzerland: WHO Bulletin Technical Report Series, 1985. p. 57.
23. WHO. Tropical diseases: progress in reerch 1989-90. Tenth programme Report of the UNDP/WORLD BANK/WHO special programme for research and Training in Tropical Diseases; 1991.

24. WHO. The control of schistosomiasis. Second report of WHO expert committee. World Health Organ Tech Rep Ser. 1993;830:1-86. 